\title{
Evaluación de la Adaptación al Divorcio-Separación: Propiedades Psicométricas del CAD-S en el Contexto Chileno
}

\author{
Evaluating Adaptation to Divorce-Separation: \\ Psychometric Properties of the CAD-S in the Chilean Context
}

\author{
Karla Tay-Karapas \\ Universidad Católica del Norte y Universidad del País Vasco
}

\author{
Mónica Guzmán-González \\ Universidad Católica del Norte
}

\author{
Sagrario Yárnoz-Yaben \\ Universidad del País Vasco
}

\begin{abstract}
El divorcio-separación supone un proceso de adaptación al nuevo estatus, dada la serie de cambios que deben afrontarse en distintos ámbitos de la vida. En tal sentido, contar con instrumentos adaptados a la realidad chilena que permitan evaluar la adaptación al divorcio y la separación es un paso necesario para comprender este proceso. El presente estudio tuvo como objetivo examinar las propiedades psicométricas de la versión chilena del Cuestionario de Ajuste al Divorcio y la Separación (CAD-S, Yárnoz Yaben \& Comino González, 2010). La muestra, no probabilística de conveniencia, estuvo compuesta por 348 progenitores, divorciados/as-separados/as provenientes de 4 ciudades de Chile. Los análisis dan cuenta de la idoneidad de un instrumento de 18 ítems y un análisis factorial confirmatorio apoya la estructura de 4 factores correlacionados, al igual que la versión desarrollada en España. Los coeficientes de consistencia interna de los factores, evaluados con el coeficiente Omega, fueron aceptables para las subescalas de dificultades psicológicas, conflictos con la expareja y disposición a la coparentalidad, con valores de $0,92,0,74$ y 0,83 respectivamente; mientras que para consecuencias negativas en los hijos fue 0,65 , siendo un valor menos aceptable. Respecto de la evidencia de validez basada en la relación con otras variables, se obtuvieron correlaciones entre el ajuste al divorcio-separación y medidas de salud mental: depresión, ansiedad y estrés (DASS-21), y satisfacción con la vida (SWLS). La escala presenta evidencia de validez para su uso en la población estudiada.
\end{abstract}

Palabras clave: adaptación al divorcio, coparentalidad, adaptación de instrumento, propiedades psicométricas.

\begin{abstract}
Divorce-separation entails a process of adaptation to one's new status, given the series of changes that must be faced in several areas of life. In this regard, having instruments suited to the Chilean context that make it possible to evaluate adaptation to divorce and separation is a necessary step for understanding this process. The objective of this study was to examine the psychometric properties of the Chilean version of the Divorce and Separation Adjustment Questionnaire (CAD-S, Yárnoz Yaben \& Comino González, 2010). The non-probability convenience sample used was composed of 348 divorced-separated parents from 4 cities in Chile. The analyses performed show the suitability of an 18-item instrument, while a confirmatory factor analysis supports a structure of 4 correlated factors, as does the version developed in Spain. The internal consistency coefficients of the factors, evaluated with the Omega coefficient, were acceptable for the subscales of psychological difficulties, conflicts with ex-partner, and attitude toward co-parenting, with values of $0.92,0.74$, and 0.83 respectively; however, a less acceptable value was obtained for negative consequences in children (0.65). Regarding the validity evidence based on relationships with other variables, correlations were obtained between adjustment to divorce-separation and mental health measures: depression, anxiety and stress (DASS-21), and satisfaction with life (SWLS). The scale has validity evidence for its use in the studied population.
\end{abstract}

Keywords: adaptation to divorce, co-parenting, instrument adaptation, psychometric properties.

Con el correr de los años, la sociedad ha experimentado grandes cambios y las instituciones sociales viven

Karla Tay-Karapas, Escuela de Psicología, Universidad Católica del Norte, Antofagasta, Chile y Facultad de Psicología, Universidad del País Vasco, San Sebastián, España; Mónica Guzmán-González, Escuela de Psicología, Universidad Católica del Norte, Antofagasta, Chile; Sagrario Yárnoz-Yaben, Facultad de Psicología, Universidad del País Vasco, San Sebastián, España.

Esta investigación ha sido financiada por el Fondo Nacional de Desarrollo Científico y Tecnológico del Gobierno de Chile, proyecto FONDECYT 1150044, concedido a la segunda autora.

La correspondencia relativa a este artículo debe ser dirigida a Karla Tay-Karapas, Escuela de Psicología, Universidad Católica del Norte, Avda. Angamos 0610, Antofagasta, Chile. E-mail: ktay@ucn.cl

Nota: Las autoras asumen la importancia de la distinción lingüística de género; sin embargo, para facilitar la lectura del texto, en adelante esta será obviada. 
un acelerado proceso de transformación, frente al cual la estructura familiar no ha estado ajena. A nivel internacional se ha reportado que el divorcio es un fenómeno frecuente (Amato, 2010), y en Chile el panorama no es muy diferente, observándose también un incremento en los divorcios (United Nations, 2011).

El divorcio es concebido como la disolución del eje conyugal y constituye un proceso complejo, que se inicia cuando los vínculos afectivos que unían a los miembros de la pareja empiezan a debilitarse, previo a concretar la ruptura física (Amato, 2010). La literatura plantea posiciones discrepantes respecto del efecto que tiene el divorcio-separación en las personas que lo experimentan. Algunos investigadores señalan que el divorcio representaría un evento altamente estresante, con consecuencias negativas en diferentes esferas de la vida de los individuos y su entorno (Amato, 2010; Yárnoz-Yaben et al., 2018). Otros afirman que el divorcioseparación podría ser un evento liberador, pues en la actualidad es un fenómeno socialmente normativo, siendo sus dificultades generalmente transitorias y limitadas (Leopold, 2018). Finalmente, hay quienes señalan que las personas divorciadas podrían experimentar ambas vivencias, estrés y liberación, así como también la posibilidad de que la experiencia varíe con el transcurso del tiempo (Rollie \& Duck, 2006). En tal sentido, divorcio-separación no sería un evento puntual, sino un proceso que consta de diversas etapas a través de las cuales la persona divorciada transita en su camino hacia la reorganización vital y afectiva (Rollie $\&$ Duck, 2006). Excepción a dicho proceso ocurre en el caso de los divorcios considerados destructivos o difíciles, en los que dicha reorganización no ocurre, incluso con el paso del tiempo (Martínez-Pampliega et al., 2015).

Con independencia de las diferentes perspectivas respecto del impacto del divorcio-separación, existe consenso en que la disolución del eje conyugal involucra un proceso de cambios sociales y emocionales a los que las personas divorciadas deben acomodarse, lo cual es conceptualizado como ajuste o adaptación al divorcio y la separación (Amato, 2000). En efecto, el divorcio-separación implica la reorganización de diferentes roles y de la estructura familiar, la adaptación a una nueva vida y la superación del duelo de la pérdida de la pareja (Yárnoz-Yaben, 2017).

En la conceptualización de la adaptación al divorcio y la separación existen diversos énfasis respecto de los desafíos involucrados. Así, por ejemplo, Sweeper y Halford (2006) señalan que existen tres desafíos específicos para el logro del ajuste psicológico post divorcio: la aceptación de la pérdida de la expareja, la aceptación de la merma de las redes sociales asociadas a la expareja y la relación como padres divorciados, que puede manifestarse como coparentalidad negativa, entendida como problemas en el ejercicio de la coparentalidad entre los progenitores. En otras aproximaciones el foco está puesto principalmente en aquellos aspectos vinculados al ejercicio de la coparentalidad como un elemento clave de la adaptación al divorcioseparación (Lamela, Figueiredo, Bastos \& Feinberg, 2016; Yárnoz-Yaben, 2010a). Tal énfasis se fundamenta en antecedentes que dan cuenta de que la adaptación al divorcio de los progenitores y el establecimiento de un plan de coparentalidad facilitarían la adaptación de los hijos al divorcio (Yárnoz-Yaben, 2010a; YárnozYaben, Comino \& Garmendia, 2012), incluso cuando estos son adultos (Yárnoz-Yaben \& Garmendia, 2016). Desde esta posición, se sostiene que la separación de los cónyuges no siempre traerá consigo el fin de las tensiones o conflictos con la expareja y el hecho de que persistan tiene importantes implicancias, no solo en el bienestar individual, sino también en la vida y desarrollo de los hijos en medidas de bienestar subjetivo y presencia de afecto negativo, entre otros aspectos (Elam, Sandler, Wolchik, Tein \& Rogers, 2019; McHale \& Lindahl, 2011; Stallman \& Ohan, 2016; Yárnoz-Yaben, 2013; Yárnoz-Yaben \& Garmendia, 2016). De allí, entonces, que el ejercicio de la coparentalidad postdivorcio se le considere un elemento clave.

El ajuste al divorcio-separación se ha asociado, también, a una serie de consecuencias sobre la salud mental y la satisfacción con la vida de las propias personas divorciadas (García \& Ilabaca Martínez, 2013; Yárnoz-Yaben et al., 2018; Yárnoz-Yaben \& Garmendia 2016). Respecto de la salud mental, se han detectado mayores índices de ansiedad, depresión, baja autoestima y riesgo suicida en personas divorciadas o separadas (Amato, 2010; Hughes \& Waite, 2009; Yip, Yousuf, Chan, Yung \& Wu, 2015). En la misma línea, otras investigaciones señalan que tanto las mujeres como los hombres divorciados evidencian menores indicadores de bienestar que las personas solteras, casadas o viudas (Doré \& Bolger, 2018; Roberson, Norona, Lenger \& Olmstead, 2018) y que los hombres solteros, divorciados o viudos de mediana edad tienen el doble de probabilidad de morir temprano que sus equivalentes casados (Bulanda, Brown \& Yamashita, 2016). Asimismo, se ha concluido que las personas divorciadas presentan menores niveles de satisfacción con la vida (Forste \& Heaton, 2004; Lucas, 2005) y que esta se encuentra estrechamente relacionada con la adaptación al divorcio, influyendo de manera positiva, a su vez, en la adaptación al divorcio de los hijos e hijas, a través del nivel de conflicto, las actitudes y las habilidades, y la implicación materna y, sobre todo, paterna con los hijos e hijas (Yárnoz Yaben, 2010b). El conjunto de estos antecedentes releva, entonces, la importancia que 
tiene la adaptación al divorcio, tanto para los adultos divorciados como para sus hijos y la dinámica de todo el grupo familiar.

En la literatura sobre la adaptación al divorcio encontramos que la mayor parte de los instrumentos para evaluarla provienen principalmente de población anglosajona, tales como la Fisher Divorce Adjustment Scale (FDAS; Fisher, 1978), el Divorce Adjustment Inventory-Revised (Portes, Smith \& Brown, 2000) y la Psychological Adjustment to Separation Test (PAST; Sweeper \& Halford, 2006). Si bien dos de estos instrumentos han sido validados para su uso en contexto chileno, el FDAS (Guzmán-González, Garrido, Calderón, Contreras \& Rivera, 2017), y el PAST (Guzmán-González, Barraza, Bastías, Bustmante \& Ojeda, 2018), el foco principal de estas escalas está puesto sobre el proceso emocional e individual de adaptación al divorcio.

Uno de los instrumentos desarrollados en población hispanoparlante y cuyo aporte diferencial es que proporciona información sobre un espectro más amplio de la dinámica familiar postdivorcio es el Cuestionario de Adaptación al Divorcio y la Separación (CAD-S; Yárnoz Yaben \& Comino González, 2010), que está diseñado específicamente para ser respondido por progenitores divorciados-separados. Esta escala pone especial énfasis en los aspectos vinculados a la percepción del impacto que la separación tiene sobre la familia y los hijos en particular, en coherencia con la aproximación que considera clave la coparentalidad en el proceso de adaptación al divorcio-separación. El CAD-S es, entonces, una escala particularmente útil para su uso en el contexto de familias con hijos menores que transitan por procesos de separación. La escala, diseñada en España, se desarrolló sobre una base inicial de 50 ítems que cubren diferentes aspectos relativos a la adaptación al divorcio-separación, basados en cuestionarios previos. Tras los análisis estadísticos, la escala quedó compuesta por 20 ítems distribuidos en cuatro factores o dimensiones: (a) Dificultades psicológicas en la adaptación al divorcio-separación, que da cuenta de enojo/rabia hacia la expareja, dificultad para aceptar la separación y un interés exagerado por las actividades que desarrolla la expareja en la actualidad; (b) Conflicto con la expareja, que retrata conflictos explícitos con la expareja: peleas, discordias, desavenencias; (c) Disposición a la coparentalidad, que dice relación con la colaboración y apoyo mutuo en temas referentes a la crianza y cuidado de los hijos y (d) Consecuencias negativas de la separación para los hijos, que da cuenta de la creencia de la madre o padre que la separación ha perjudicado a sus hijos, ya sea en términos emocionales o sociales (económicos).

Si bien el CAD-S ya se encuentra en castellano, la relevancia de validar el uso de las puntuaciones en el contexto chileno se sustenta en la noción de que la utilización de test o pruebas psicológicas creadas en diferentes contextos culturales requiere de un proceso de adaptación que incluye su traducción o posibles cambios a nivel semántico, aunque se realicen sobre la base de un mismo idioma (Muñiz, Elosua \& Hambleton, 2013).

Considerando los antecedentes expuestos, el objetivo del presente estudio fue evaluar las propiedades psicométricas del CAD-S (Yárnoz Yaben \& Comino González, 2010), con el propósito de contribuir al desarrollo de una versión del instrumento válida y confiable para su uso en población de progenitores chilenos divorciados-separados. Para el logro de los objetivos, se analizó su estructura factorial, se evaluó la consistencia interna y la evidencia de validez basada en la relación con otras variables.

Las hipótesis que guían el presente estudio son las siguientes: (a) El CAD-S Chile posee la estructura de cuatro dimensiones propuestas en su versión original, conformada por: dificultades psicológicas, conflicto con la expareja, disposición a la coparentalidad y consecuencias negativas en los hijos; (b) Existe asociación entre las dimensiones del CAD-S Chile e indicadores de salud mental, específicamente, mientras más positiva es la adaptación al divorcio-separación, hay menores síntomas de depresión, ansiedad y estrés y (c) Existe asociación entre las dimensiones del CAD-S Chile y la satisfacción con la vida, específicamente, mientras mejor es la adaptación al divorcio-separación, hay mayores niveles de satisfacción vital.

\section{Participantes}

\section{Método}

En este estudio participaron 348 progenitores chilenos divorciados o separados, distribuidos en 234 mujeres y 114 hombres, provenientes de cuatro ciudades de distintas zonas geográficas de Chile: Antofagasta (norte), Santiago y Talca (centro) y Concepción (sur). Fueron reclutados mediante un muestreo no probabilístico de conveniencia. Para su selección se utilizaron dos vías: contacto directo y contacto a través de redes sociales. 
El promedio de edad fue de 40,40 años $(D E=11,40)$. Respecto del nivel educacional, un 52,9\% tenía estudios superiores (universitarios o técnicos), un $29,5 \%$, estudios secundarios, mientras que un $12,2 \%$ refirió poseer estudios inferiores a estos.

El promedio de duración de la relación con la expareja fue de 11,55 años $(D E=7,89)$. El tiempo promedio transcurrido desde la separación a la actualidad fue de 3,85 años $(D E=3,42)$. Finalmente, con relación a la custodia de los hijos, un $87,3 \%$ estaba a cargo de la madre y un $14,7 \%$, del padre.

\section{Instrumentos}

Cuestionario sociodemográfico. Se confeccionó un cuestionario que permitiera recopilar los datos sociodemográficos de la muestra, tales como edad, sexo, nivel educacional, ciudad de residencia y estado civil. Además, se incluyó la caracterización de variables como duración de la relación con la expareja, tiempo transcurrido desde el divorcio-separación, situación de pareja actual, presencia de hijos, número de hijos y edades de cada uno.

Cuestionario de Adaptación al Divorcio-Separación (CAD-S; Yárnoz Yaben \& Comino González, 2010). Este cuestionario de autorreporte, desarrollado en España, se utiliza para evaluar la adaptación al divorcio-separación del grupo familiar en conjunto, ocupando a uno de los progenitores como informante. Consta de 20 ítems que se contestan en una escala Likert con respuestas que van desde 1 (totalmente en desacuerdo) a 5 (totalmente de acuerdo). Está organizado en cuatro dimensiones: (a) Dificultades psicológicas, compuesto por 6 ítems (“Creo que nunca voy a superar la separación”), (b) Conflicto con la expareja, compuesto por 3 ítems ("Desde el divorcio mi expareja y yo nos hemos gritado el uno al otro"), (c) Disposición a la coparentalidad, compuesto por 5 ítems ("Mi expareja es una ayuda para mí en la crianza de nuestros hijos") y (d) Consecuencias negativas en los hijos, compuesto por 6 ítems ("Nuestra situación financiera después del divorcio nos ha obligado a cambiar de casa"). Tres de las cuatro dimensiones se refieren a aspectos que dificultan la adaptación al divorcio mientras que sólo la disposición a la coparentalidad se puede considerar como factor de un buen ajuste, por lo tanto, para tener una visión general acerca de la adaptación al divorcio-separación, se puede obtener un índice positivo o negativo utilizando las puntuaciones obtenidas y aplicando la siguiente fórmula: Ajuste = Coparentalidad - (Dif. Psicológicas + Conflicto + Cons. Hijos) /3. Así una puntuación de signo positivo revelará un ajuste positivo a la situación de divorcio, mientras que una puntuación negativa mostrará un ajuste negativo (Yárnoz Yaben \& Comino González, 2010).

El coeficiente de consistencia interna basado en alfa de Cronbach fue 0,77 para la escala total; 0,78 para Dificultades psicológicas, 0,81 para Conflicto con la expareja, 0,72 para Disposición a la coparentalidad y 0,65 para Consecuencias negativas en los hijos (Yárnoz Yaben \& Comino González, 2010). En lo que respecta a la validez del instrumento, el análisis de componentes principales mostró una estructura de cuatro factores integrados por 20 ítems que presentan saturaciones claras y una gran coherencia teórica, constatándose que el CAD-S evalúa aspectos esenciales que reflejan el nivel de adaptación del grupo familiar a la situación de divorcio (Yárnoz Yaben \& Comino González, 2010). Asimismo, posee una adecuada validez de constructo, donde las puntuaciones obtenidas por los progenitores en función de su ajuste al divorcio correlacionan de manera estadísticamente significativa con variables relevantes asociadas al bienestar psicológico de los distintos componentes del grupo familiar. Encontrándose que la satisfacción vital (SWLS) es más alta en los progenitores con un mayor nivel de ajuste al divorcio, y los índices de problemas de conducta en los hijos (CBCL) son más bajos en aquellos grupos familiares donde se da un ajuste positivo al divorcio (Yárnoz Yaben \& Comino González, 2010).

Escala Abreviada de Depresión, Ansiedad y Estrés (DASS-21; Lovibond \& Lovibond, 1995). Esta escala está validada en Chile por Román, Vinet y Alarcón (2014). Este instrumento se utiliza para evaluar salud mental y está compuesto de 21 ítems contestados en una escala Likert con respuestas que van desde 0 (No describe en nada lo que me ocurrió o sentí durante la semana) a 3 (Sí, esto me pasó mucho o casi siempre). Está organizado en tres dimensiones: (1) Depresión, compuesta por 7 ítems ("Me sentí triste y deprimido"), (2) Ansiedad, compuesta por 7 ítems ("Me di cuenta que tenía la boca seca"), y (3) Estrés, compuesta por 7 ítems ("Sentí que estaba muy Nervioso"), donde a mayores puntajes, mayor presencia de sintomatología depresiva, ansiosa o estrés. Para el instrumento de Román, Vinet y Alarcón (2014), la consistencia interna basada en alfa de Cronbach fue 0,85 para la escala de Depresión, 0,72 para la escala de Ansiedad y 0,79 para la escala de Estrés. En el presente estudio los alfas obtenidos en Depresión, Ansiedad y Estrés fueron 0,89, 
0,87 y 0,90, respectivamente. Respecto de la validez convergente y discriminante, se utilizó el coeficiente de correlación de Pearson entre las escalas del DASS-21 con el Inventario de Depresión de Beck (BDI) y el Inventario de Ansiedad de Beck (BAI). La escala de Depresión del DASS-21 mostró una correlación estadísticamente significativa con el BDI mientras que con el BAI fue significativa pero menor. La escala de Ansiedad del DASS-21 mostró una correlación estadísticamente significativa con el BAI, mientras que con el BDI esta correlación fue significativa pero menor. La escala de Estrés del DASS-21 mostró una correlación estadísticamente significativa tanto con el BAI como con el BDI siendo esta última ligeramente menor (Román, Vinet \& Alarcón, 2014).

Escala de Satisfacción con la Vida (SWLS; Diener, Emmons, Larsen \& Griffin, 1985). Está validada en Chile por Vera-Villarroel, Urzúa, Pavez, Celis-Atenas y Silva (2012). Este instrumento mide el nivel de satisfacción vital y consta 5 ítems ("Estoy satisfecho con mi vida") contestados en una escala Likert con respuestas que van desde 1 (No, en absoluto) a 7 (Sí, totalmente), agrupados en una dimensión; donde a mayores puntajes, se reflejan mejores niveles de satisfacción vital. El coeficiente de consistencia interna basado en alfa de Cronbach fue 0,82 (Vera-Villarroel, Urzúa, Pavez, Celis-Atenas y Silva, 2012). Para este estudio el índice de consistencia interna fue 0,80. Respecto a la validez discriminante, la SWLS se correlaciona inversamente con valores obtenidos en la Escala de Regulación Emocional (DERS). Mientras que en relación a la validez convergente, la SWLS se correlaciona con la Calidad de Vida Global (WHOQOLBREF) y los diferentes dominios de manera directa y significativa (Vera-Villarroel, Urzúa, Pavez, CelisAtenas y Silva, 2012).

\section{Procedimiento}

El estudio contó con la aprobación del comité de ética de la Universidad Católica del Norte. Se solicitó autorización a las autoras del CAD-S para la adaptación del instrumento en población chilena.

Una vez conseguido el consentimiento de las autoras, se solicitó a un equipo conformado por tres psicólogos la revisión del instrumento mediante la lectura de los 20 reactivos y su posterior evaluación semántica, con la finalidad de incorporar sus sugerencias respecto de las representaciones formales que fueran propias de España, para sustituirlas por expresiones propias de Chile. De este análisis, el contenido del ítem 7 fue reformulado: "Estoy enfadado/a hacia mi expareja" se cambió por "Estoy enojado/a con mi expareja", dado que enojo es una expresión más acorde al contexto chileno que la palabra enfado.

Luego, se proporcionó información a cada participante sobre los objetivos y naturaleza del estudio, enfatizando la confidencialidad de sus datos, el resguardo de la información y la voluntariedad de su participación. Finalmente se procedió a la firma del consentimiento informado, en formato papel o digital, según correspondía.

A los participantes cuyo contacto fue directo, los instrumentos fueron aplicados en formato papel por un equipo de encuestadores previamente capacitados y a los contactados a través de redes sociales, se difundió el cuestionario a través de un enlace electrónico.

\section{Análisis de Datos}

Para el examen de la estructura factorial del CAD-S Chile se llevó a cabo un análisis factorial confirmatorio (AFC) con el programa Mplus 7.4 (Muthén \& Muthén, 2017), dado que su estructura teórica ya era conocida (Yárnoz Yaben \& Comino González, 2010). Para ello se extrajo de la muestra total una submuestra aleatoria de aproximadamente el $40 \%$ de la misma $(N=129)$, en la que se testeó la propuesta de cuatro dimensiones, para luego revalidarla en el resto de la muestra $(N=219)$. Se permitió la correlación entre los factores y se fijó en 1 la carga de cada factor. Se ocupó la matriz de correlaciones policóricas para los análisis y los mínimos cuadrados ponderados con media y varianza ajustada como método de estimación, al considerar las variables como ordinales. Se analizaron datos completos, dado que no hubo respuestas omitidas.

Se incluyeron como índices de ajuste del modelo $\chi^{2}$ y el índice comparativo de Bentler-Bonett (CFI) y el índice de Tucker Lewis (TLI), con valores sobre 0,95 considerados como aceptables, así como la raíz del error cuadrático medio de aproximación (RMSEA), en el que se esperaban valores inferiores a 0,06 (Hu \& Bentler, 1999). 
Luego de ello, se procedió a calcular los coeficientes de consistencia interna de las subescalas del cuestionario, medidos a través del coeficiente Omega.

Finalmente, para la evaluación de la validez asociada a criterio, se examinó la relación entre las dimensiones del CAD-S Chile y medidas de satisfacción con la vida (SWLS) y salud mental (DASS-21), mediante el coeficiente de correlación de Pearson.

\section{Resultados}

\section{Estructura Factorial del CAD-S Chile}

El modelo original evidenció un pobre ajuste a los datos, $\chi^{2}(164, N=129)=391,12, p<0,001 ; \chi^{2} / g l=2,38$; $\mathrm{CFI}=0,92 ; \mathrm{TLI}=0,90 ; \mathrm{RMSEA}=0,104,90 \% \mathrm{CI}[0,090,0,117]$.

$\mathrm{Al}$ examinar las cargas factoriales, se observó que el ítem 20 ("En general, me gustaría que mis hijos pudieran ver más a su padre/madre") no tenía carga significativa en el factor hipotetizado ni en ninguno de los otros factores de la escala, según se observó al examinar los índices de modificación. Asimismo, los índices de modificación dieron cuenta que el ítem 7 ("Estoy enojada/o con mi expareja") tenía carga en el factor original, Dificultades psicológicas, pero también en las dimensiones Conflicto con la expareja y Consecuencias negativas en los hijos.

La revisión del contenido del ítem 20 da cuenta de la expresión de un deseo, que no necesariamente se refiere a consecuencias negativas en los hijos, que es el factor al que originalmente pertenecía, ni tampoco en su redacción es claro que pueda pertenecer a alguna de las otras dimensiones. Al mismo tiempo, el ítem 7 puede reflejar dificultades psicológicas, pero también puede dar cuenta de conflictos con la expareja, lo que puede asociarse a consecuencias negativas sobre los hijos.

Tabla 1

Análisis Factorial Confirmatorio de CAD-S Chile: Cargas Factoriales Estandarizadas

\begin{tabular}{rcccc}
\hline Ítem & Factor 1 & Factor 2 & Factor 3 & Factor 4 \\
\hline 6 & 0,92 & & & \\
8 & 0,88 & & & \\
9 & 0,97 & & & \\
12 & 0,86 & & & \\
17 & 0,82 & & & \\
1 & & 0,79 & & \\
4 & & 0,90 & & \\
5 & & 0,68 & 0,54 & \\
11 & & & 0,79 & \\
14 & & & 0,82 & \\
15 & & & 0,84 & \\
16 & & & 0,79 & 0,60 \\
19 & & & & 0,66 \\
2 & & & & 0,55 \\
3 & & & & 0,43 \\
10 & & & & 0,70 \\
13 & & & & \\
18 & & & & \\
\hline
\end{tabular}

Nota. Factor 1 = Dificultades psicológicas; Factor $2=$ Conflicto con la expareja; Factor $3=$ Disposición a la coparentalidad; Factor $4=$ Consecuencias negativas en los hijos. Se presentan los números originales de los ítems de la versión española del CAD-S. Por tal motivo, no aparecen los reactivos 7 y 20 , que fueron suprimidos en la versión chilena. $N=129$. 
En consecuencia, considerando ambos criterios, teóricos y estadísticos, se re-estimó un segundo modelo, excluyendo los ítems problemáticos (7 y 20). Este nuevo modelo evidenció un mejor ajuste a los datos, $\chi^{2}(129$, $N=129)=223,75, p<0,001 ; \chi^{2} / \mathrm{gl}=1,73 ; \mathrm{CFI}=0,96 ;$ TLI $=0,96 ;$ RMSEA $=0,075,90 \%$ CI $[0,059,0,092]$.

La inspección de las cargas factoriales de este nuevo modelo revela que todas fueron significativas, de magnitudes adecuadas $(>0,40)$ y en las direcciones esperadas, con valores entre 0,43 y 0,97 (ver Tabla 1), replicándose la estructura de cuatro factores propuesta en el instrumento original.

Asimismo, se identificó correlación entre todos los factores $(p<0,05)$, con valores que fluctuaron entre 0,19 y 0,78 (ver Tabla 2 ).

Tabla 2

Correlaciones entre las Subescalas del CAD-S Chile en la Muestra $1(N=129)$ y en la Muestra $2(N=219)$

\begin{tabular}{lcccc}
\hline \multicolumn{1}{c}{ Subescala } & 1 & 2 & 3 & 4 \\
\hline 1. Dificultades psicológicas & - & $0,36^{* *}$ & $0,18^{* *}$ & $0,59^{* *}$ \\
2. Conflicto con la expareja & $0,34^{*}$ & - & $-0,20^{* *}$ & $0,64^{* *}$ \\
3. Disposición a la coparentalidad & $0,19^{*}$ & $-0,34^{* *}$ & - & $-0,14^{*}$ \\
4. Consecuencias negativas en los hijos & $0,62^{*}$ & $0,78^{* *}$ & $-0,30^{* *}$ & - \\
\hline
\end{tabular}

Nota. ${ }^{*} p<0,05 * * p<0,01$. Correlaciones para la muestra 1 se encuentran bajo la diagonal y sobre la diagonal para la muestra 2 .

Tabla 3

Reanálisis Factorial Confirmatorio CAD-S Chile: Cargas Factoriales Estandarizadas

\begin{tabular}{rcccc}
\hline Ítem & Factor 1 & Factor 2 & Factor 3 & Factor 4 \\
\hline 6 & 0,93 & & & \\
8 & 0,90 & & & \\
9 & 0,95 & & & \\
12 & 0,89 & & & \\
17 & 0,87 & & & \\
1 & & 0,76 & & \\
4 & & 0,84 & & \\
5 & & 0,64 & 0,56 & \\
11 & & & 0,87 & \\
14 & & & 0,79 & \\
15 & & & 0,92 & \\
16 & & & 0,64 & 0,59 \\
19 & & & & 0,67 \\
2 & & & & 0,58 \\
3 & & & & 0,55 \\
10 & & & & 0,70 \\
13 & & & \\
18 & & & & \\
\hline
\end{tabular}

Nota. Factor 1 = Dificultades psicológicas; Factor $2=$ Conflicto con la expareja; Factor $3=$ Disposición a la coparentalidad; Factor $4=$ Consecuencias negativas en los hijos. $N=255$.

Posteriormente, y a fin de evaluar la replicabilidad y ajuste de este modelo con los dos reactivos menos que se proponen para la versión chilena, se realizó un nuevo AFC con la segunda parte de la muestra total $(N=$ 219). El ajuste del modelo con 18 reactivos evidenció un ajuste aceptable a los datos, $\chi^{2}(129, N=219)=$ 256,149, $p<0,001 ; \chi^{2} / \mathrm{gl}=1,99$; CFI = 0,98; TLI = 0,98; RMSEA = 0,062, 90\% CI [0,051, 0,073]. A nivel de 
ajuste local, las cargas factoriales fueron significativas, adecuadas en magnitud $(>0,40)$ y en las direcciones esperadas, con valores entre 0,55 y 0,95 (ver Tabla 3 ). Asimismo, se identificó que todos los factores estuvieron correlacionados $(p<0,05)$, con valores fluctuantes entre -0,14 y 0,64 (ver Tabla 2 ).

\section{Consistencia Interna}

Los coeficientes de consistencia interna fueron $0,92,0,74,0,83$ y 0,65 para las subescalas de Dificultades psicológicas, Conflictos con la expareja, Disposición a la coparentalidad y Consecuencias negativas en los hijos, respectivamente.

\section{Evidencia de Validez Basada en la Relación con Otras Variables}

Finalmente, se evaluó la correlación entre las cuatro dimensiones del CAD-S Chile e indicadores de salud mental (DASS-21) y satisfacción con la vida (SWLS). En cuanto al factor Dificultades psicológicas y al factor Consecuencias negativas en los hijos, se observan correlaciones con todas las dimensiones del DASS-21 (Depresión, Ansiedad y Estrés) y con satisfacción con la vida (SWLS) en las direcciones esperadas. Para el factor Conflicto con la expareja se evidencian correlaciones con las dimensiones Depresión y Estrés del DASS21. Por el contrario, para el factor Disposición a la coparentalidad no se advierten relaciones con ninguna de las dimensiones del DASS-21 ni con el SWLS (ver Tabla 4).

Tabla 4

Correlaciones entre las Subescalas del CAD-S Chile e Indicadores de Salud Mental y Satisfacción con la Vida

\begin{tabular}{lcccc}
\hline \multicolumn{1}{c}{ Indicador } & $\begin{array}{c}\text { Dificultades } \\
\text { psicológicas }\end{array}$ & $\begin{array}{c}\text { Conflicto con la } \\
\text { expareja }\end{array}$ & $\begin{array}{c}\text { Disposición a la } \\
\text { coparentalidad }\end{array}$ & $\begin{array}{c}\text { Consecuencias } \\
\text { negativas en los hijos }\end{array}$ \\
\hline Depresión & $0,30^{* *}$ & $0,13^{*}$ & $-0,09$ & $0,17^{* *}$ \\
Ansiedad & $0,28^{*}$ & 0,10 & $-0,08$ & $0,16^{* *}$ \\
Estrés & $0,29^{*}$ & $0,16^{* *}$ & $-0,07$ & $0,16^{* *}$ \\
Satisfacción & $-0,28^{*}$ & $-0,10$ & $-0,08$ & $0,26^{* *}$ \\
con la vida & & & & \\
\hline
\end{tabular}

Nota. ${ }^{*} p<0,05 * * p<0,01$

\section{Discusión y Conclusiones}

El objetivo del presente estudio fue evaluar las propiedades psicométricas del CAD-S para su uso en el contexto chileno. De los análisis realizados, se desprende que la versión chilena del CAD-S, compuesta por 18 ítems de los 20 presentes en la versión española, se presenta como un instrumento con adecuadas propiedades psicométricas para su uso.

En primer lugar y respecto de la primera hipótesis relacionada con la estructura factorial del CAD-S, se confirma lo propuesto, dado que la escala se organiza en cuatro dimensiones: Dificultades psicológicas, Conflicto con la expareja, Disposición a la coparentalidad y Consecuencias negativas en los hijos, tal como se había propuesto en el desarrollo original de la versión española. La puesta a prueba de la estructura factorial de la escala mediante una técnica estadística más potente y que controla el error de medida, como es el AFC, refuerza y le da más sustento a los hallazgos reportados en la versión española. Cabe mencionar, no obstante, que se decidió remover dos reactivos con base en criterios sustantivos y estadísticos, dado que ambos reactivos incluidos podrían ser reflejo también de las otras dimensiones, como conflictos con la ex pareja y consecuencias negativas para las y los hijos. Además y de acuerdo a lo que habría que esperar, los factores se asociaron entre sí en magnitudes bajas y moderadas, lo que indica que son dimensiones independientes, pero correlacionadas entre sí.

Asimismo, casi todos los coeficientes de consistencia interna de las subescalas se ubicaron dentro de rangos aceptables. Si bien la subescala de Consecuencias negativas en los hijos obtuvo un valor menor al aceptable, ello podría tener relación con la baja cantidad de reactivos que componen dicha subescala, lo que sugiere cautela respecto de su uso. 
Respecto de la segunda y la tercera hipótesis, relativas a la evidencia de validez basada en la relación con otras variables, se encontró que a mayor percepción de dificultades psicológicas y de consecuencias negativas en los hijos se reportan mayores síntomas de ansiedad, depresión y estrés y menores niveles de satisfacción vital. Similar patrón de resultados se observó en el caso de la dimensión Conflictos con la expareja, que se asoció a mayores síntomas de ansiedad y estrés, pero no con depresión ni satisfacción vital. Tal resultado puede explicarse en términos de que el conflicto con quien ya no se tiene un vínculo amoroso, pero sí uno de carácter instrumental, puede asociarse a un aumento de la tensión, pero no necesariamente impactar el estado de ánimo ni la apreciación de la vida. En su conjunto, estos resultados son coherentes con evidencia previa que vincula el ajuste al divorcio a mejores índices de salud mental (e.g., Doré \& Bolger, 2018; Roberson et al., 2018). Respecto de la disposición a la coparentalidad, no se identificó asociación con ninguna de las variables antes mencionadas. La inspección de los ítems que componen esta escala dan cuenta del acuerdo en cuestiones de tipo instrumental y de apoyo en la crianza de los hijos, lo cual no necesariamente tendría relación con los indicadores de salud mental y satisfacción vital. Futuros estudios podrían explorar este aspecto en mayor profundidad.

Pese a que los resultados obtenidos apuntan a que el CAD-S en su versión chilena sería un instrumento adecuado para la evaluación del ajuste al divorcio y la separación en padres divorciados, el presente estudio posee una serie de limitaciones que deben ser consideradas. En primer lugar, es necesario tener cautela respecto de la generalización de estos hallazgos, dado el carácter no probabilístico de la muestra y a que ella se encuentra conformada mayoritariamente por personas con niveles medios y altos de educación. Futuros estudios podrían examinar las propiedades de la escala en muestras más heterogéneas. En segundo lugar, el tamaño de la muestra no permitió evaluar la invarianza de la escala entre hombres y mujeres, tarea que queda pendiente para futuros estudios.

Por último y a modo de conclusión, es importante considerar que si bien el CAD-S Chile es un instrumento cuyas puntuaciones permiten dar cuenta de la adaptación de progenitores chilenos al divorcio o separación, los resultados provenientes de su aplicación deben ser interpretados responsablemente, pues no se considera como un predictor de mejores o mayores habilidades parentales, por lo que no se aconseja su uso como criterio exclusivo para concluir la idoneidad parental.

\section{Referencias}

Amato, P. R. (2000). The consequences of divorce for adults and children. Journal of Marriage and Family, 62, $1269-1287$. https://doi.org/10.1111/j.1741-3737.2000.01269.x

Amato, P. R. (2010). Research on divorce: Continuing trends and new developments. Journal of Marriage and Family, 72, 650-666. https://doi.org/10.1111/j.1741-3737.2010.00723.x

Bulanda, J. R., Brown, J. S. \& Yamashita, T. (2016). Marital quality, marital dissolution, and mortality risk during the later life course. Social Science \& Medicine, 165, 119-127. https://doi.org/10.1016/j.socscimed.2016.07.025

Diener, E., Emmons, R. A., Larsen, R. J. \& Griffin, S. (1985). The Satisfaction with Life Scale. Journal of Personality Assessment, 49, 71-75. https://doi.org/10.1207/s15327752jpa4901_13

Doré, B. \& Bolger, N. (2018). Population- and individual-level changes in life satisfaction surrounding major life stressors. Social Psychological and Personality Science, 9, 875-884. https://doi.org/10.1177\%2F1948550617727589

Elam, K. K., Sandler, I., Wolchik, S. A., Tein, J.-Y., \& Rogers, A. (2019). Latent profiles of postdivorce parenting time, conflict, and quality: Children's adjustment associations. Journal of Family Psychology, 33(5), 499-510. https://doi.org/10.1037/fam0000484

Fisher, B. (1978). Facilitator's manual for rebuilding: When your realationship ends. Boulder, CO: Family Relations Learning Center.

Forste, R. \& Heaton, T. B. (2004). The divorce generation: Well-being, family attitudes, and socioeconomic consequences of marital disruption. Journal of Divorce \& Remarriage, 41(1-2), 95-114. https://doi.org/10.1300/J087v41n01_06

García, F. E. \& Ilabaca Martínez, D. (2013). Ruptura de pareja, afrontamiento y bienestar psicológico en adultos jóvenes. Ajayu, 11(2), 42-60. Extraído de http://www.scielo.org.bo/pdf/rap/v11n2/a03.pdf

Guzmán-González, M., Barraza, M. A., Bastías, F., Bustamente, D. \& Ojeda, M. F. (2018). Propiedades psicométricas del Test de Ajuste Psicológico a la Separación en personas separadas y divorciadas chilenas. Terapia Psicológica, 36, 113-122. https://doi.org/10.4067/S0718-48082018000200113

Guzmán-González, M., Garrido, L., Calderón, C., Contreras, P. \& Rivera, D. (2017). Chilean adaptation and validation of the Fisher Divorce Adjustment Scale-Short Form. Journal of Divorce \& Remarriage, 58, 96-109. https://doi.org/10.1080/10502556.2016.1268017

Hu, L. \& Bentler, P. M. (1999). Cutoff criteria for fit indexes in covariance structure analysis: Conventional criteria versus new alternatives. Structural Equation Modeling, 6, 1-55. https://doi.org/10.1080/10705519909540118

Hughes, M. E. \& Waite, L. J. (2009). Marital biography and health at mid-life. Journal of Health and Social Behavior, 50, 344-358. https://doi.org/10.1177/002214650905000307

Lamela, D., Figueiredo, B., Bastos, A. \& Feinberg, M. (2016). Typologies of post-divorce coparenting and parental well-being, parenting quality and children's psychological adjustment. Child Psychiatry \& Human Development, 47, $716-728$. https://doi.org/10.1007/s10578-015-0604-5

Leopold, T. (2018). Gender differences in the consequences of divorce: A study of multiple outcomes. Demography, 55, 769-797. https://doi.org/10.1007/s13524-018-0667-6 
Lovibond, P. F. \& Lovibond, S. H. (1995). The structure of negative emotional states: Comparison of the Depression Anxiety Stress Scales (DASS) with the Beck depression and anxiety inventories. Behaviour Research and Therapy, 33, 335-343. Extraído de https://doi.org/10.1016/0005-7967(94) 00075-U

Lucas, R. E. (2005). Time does not heal all wounds: A longitudinal study of reaction and adaptation to divorce. Psychological Science, 16, 945-950. https://doi.org/10.1111/j.1467-9280.2005.01642.x

Martínez-Pampliega, A., Aguado, V., Corral, S., Cormenzana, S., Merino, L. \& Iriarte, L. (2015). Protecting children after a divorce: Efficacy of Egokitzen-An intervention program for parents on children's adjustment. Journal of Child and Family Studies, 24, 3782-3792. https://doi.org/10.1007/s10826-015-0186-7

McHale, J. P. \& Lindahl, K. M. (Eds.) (2011). Coparenting: A conceptual and clinical examination of family systems. Washington, DC: American Psychological Association. https://doi.org/10.1037/12328-000

Muñiz, J., Elosua, P. \& Hambleton, R. K. (2013). Directrices para la traducción y adaptación de los tests: segunda edición. Psicothema, 25, 151-157. https://doi.org/10.7334/psicothema2013.24

Muthén, L. K. \& Muthén, B. O. (2017). Mplus user's guide (8va ed.). Los Angeles, CA: Autores.

National Center for Health Statistics (2015). Provisional number of divorces, annulments and rate: United States, $2000-2014$. Hyattsville, MD: Autor.

Portes, P. R., Smith, T. L. \& Brown, J. H. (2000). The Divorce Adjustment Inventory-Revised: Validation of a parental report concerning children's post-custody adjustment. Journal of Divorce \& Remarriage, 33(3-4), 93-109. https://doi.org/10.1300/J087v33n03_06

Roberson, P. N. E., Norona, J. C., Lenger, K. A. \& Olmstead, S. B. (2018). How do relationship stability and quality affect wellbeing? Romantic relationship trajectories, depressive symptoms, and life satisfaction across 30 years. Journal of Child and Family Studies, 27, 2171-2184. https://doi.org/10.1007/s10826-018-1052-1

Román, F., Vinet, E. \& Alarcón, A.M. (2014). Escalas de Depresión, Ansiedad y Estrés (DASS-21): Adaptación y propiedades psicométricas en estudiantes secundarios de temuco. Revista Argentina de Clínica Psicológica, XXIII (2),179-190. Extraído de https://www.redalyc.org/articulo.oa?id=2819/281943265009

Rollie, S. S., \& Duck, S. (2006). Divorce and Dissolution of Romantic Relationships: Stage Models and Their Limitations. In M. A. Fine \& J. H. Harvey (Eds.), Handbook of divorce and relationship dissolution (p.223-240). Lawrence Erlbaum Associates Publishers. https://doi.org/10.1080/15267430802089438

Stallman, H. M. \& Ohan, J. L. (2016). Parenting style, parental adjustment, and co-parental conflict: Differential predictors of child psychosocial adjustment following divorce. Behaviour Change, 33, 112-126. https://doi.org/10.1017/bec.2016.7

Sweeper, S. \& Halford, K. (2006). Assessing adult adjustment to relationship separation: The Psychological Adjustment to Separation Test (PAST). Journal of Family Psychology, 20, 632-640. https://doi.org/10.1037/0893-3200.20.4.632

United Nations (2011). 2009-2010. Demographic Yearbook. Department of economic and social affairs. New York: Author.

Vera-Villarroel, P., Urzúa, A., Pavez, P., Celis-Atenas, K. \& Silva, J. (2012). Evaluation of subjective well-being: Analysis of the Satisfaction with Life Scale in Chilean population. Universitas Psychologica, 11, 719-727. Extraído de http://www.redalyc.org/articulo.oa?id=64724634003

Yárnoz-Yaben, S. (2010a). Hacia la coparentalidad post-divorcio: percepción del apoyo recibido de la ex pareja en progenitores divorciados españoles. International Journal of Clinical and Health Psychology, 10, 295-307. Extraído de http://www.redalyc.org/articulo.oa?id=33712250006

Yárnoz Yaben. S. (2010b). Satisfacción vital en madres y padres divorciados. Ansiedad y Estrés, 16, 163-175. Extraído de https://www.researchgate.net/publication/305496024

Yárnoz-Yaben, S. (2013). ¿Y si todo fue un error? Pensamientos negativos y ajuste al divorcio. Estudios de Psicología, 34, 185-195. https://doi.org/10.1174/021093913806751393

Yárnoz-Yaben, S. (2017). Grief due to divorce: Relationship with attachment style and effects on subjective well-being and co-parenting. Studies in Psychology, 38, 667-688. https://doi.org/10.1080/02109395.2017.1330305

Yárnoz Yaben, S. \& Comino González, P. (2010). El CAD-S, un instrumento para la evaluación de la adaptación al divorcio-separación. Psicothema, 22, 157-162. Extraído de http://www.psicothema.com/pdf/3711.pdf

Yárnoz-Yaben, S., Comino, P. \& Garmendia, A. (2012). Ajuste al divorcio de los progenitores y problemas de conducta en hijos de familias separadas. Infancia y Aprendizaje, 35, 37-47. https://doi.org/10.1174/021037012798977485

Yárnoz-Yaben, S. \& Garmendia, A. (2016). Parental divorce and emerging adults' subjective well-being: The role of "carrying messages". Journal of Child and Family Studies, 25, 638-646. https://doi.org/10.1007/s10826-015-0229-0

Yárnoz-Yaben, S., Guzmán-González, M., Comino, P., Garrido, L., Contreras, P. \& Rivera, D. (2018). Life satisfaction in divorced or separated people: A comparison between Spain and Chile. Studies in Psychology, 39, 154-178. https://doi.org/10.1080/02109395.2017.1407904

Yip, P. S. F., Yousuf, S., Chan, C. H., Yung, T. \& Wu, K. C. -C. (2015). The roles of culture and gender in the relationship between divorce and suicide risk: A meta-analysis. Social Science \& Medicine, 128, 87-94. https://doi.org/10.1016/j.socscimed.2014.12.034

Fecha de recepción: Septiembre de 2018.

Fecha de aceptación: Mayo de 2020. 